\title{
Extreme Light Infrastructure: Nuclear Physics
}

\author{
N.V. Zamfir ${ }^{\mathrm{a}}$, D. Habs ${ }^{\mathrm{b}}$, F. Negoita ${ }^{\mathrm{a}}$, D. Ursescu ${ }^{\mathrm{c}}$ \\ ${ }^{a}$ Horia Hulubei - National Institute of Physics and Nuclear Engineering, 407 Atomistilor, \\ 077125 Bucharest-Magurele, Romania \\ ${ }^{b}$ Fakultät für Physik, Ludwig Maximilians Universität, München, D-85748 Garching, Germany \\ ${ }^{c}$ National Institute for Laser, Plasma and Radiation Physics, 409 Atomistilor, \\ 077125 Bucharest-Magurele, Romania
}

\begin{abstract}
The spectacular progress of electron and heavy-ions acceleration driven by ultra-short high-power laser has opened the way for new methods of investigations in nuclear physics and related fields. On the other hand, upshifting the photon energies of a high repetition TW-class laser through inverse Compton scattering on electron bunches classically accelerated, a high-flux narrow bandwidth gamma beam can be produced. With such a gamma beam in the 1-20 MeV energy range and a two-arms 10-PW class laser system, the pillar of "Extreme Light Infrastructure" to be built in Bucharest will focus on nuclear phenomena and their practical applications. Nuclear structure, nuclear astrophysics, fundamental QED aspects as well as applications in material and life sciences, radioactive waste management and homeland security will be studied using the high-power laser, the gamma beam or combining the two. The article includes a general description of ELI-Nuclear Physics (ELI-NP) facility, an overview of the Physics Case and some details on the few, most representative proposed experiments.
\end{abstract}

Keywords: high-power ultra-short lasers pulses, monochromatic gamma beam, nuclear resonance fluorescence (NRF), positron beams

\section{INTRODUCTION}

The Extreme Light Infrastructure - Nuclear Physics (ELI-NP), one of the four pillars of ELI, is meant as a unique research facility to investigate the impact of very intense electromagnetic radiation (Extreme Light) on matter with specific focus on nuclear phenomena and their practical applications. ELI-NP is proposed to be build in Magurele, near Bucharest (Romania). From the point of view of scientific objectives as well as of technologies to be used for laser systems, it will be complementary to the other three pillars to be build in Prague (Czech Republic) - ELI-Beamlines, in Szeged (Hungary) - ELI-Attoseconds and in a location not yet decided - ELI-High Fields pillar.

ELI-NP pillar will have two kind of 'extreme light': one 'visible', generated by 10-PW class lasers, and the other one in the 'gamma' range generated by inverse Compton back-scattering of optical photons on relativistic electrons.

The objectives of ELI-NP will be manifold: to characterize the interaction of the $10 \mathrm{PW}$ class laser beams with matter using nuclear methods and tools, photonuclear reactions for basic and applied studies, fundamental physics using high intensity laser beams and/or gamma beams.

At high-power laser facilities in operation, with power levels up to $1 \mathrm{PW}$, many types of nuclear excitations and reactions have been observed following the interaction of nuclei with accelerated electrons and ions, or with emitted hard $\mathrm{X}$-rays and gamma-rays. The objective of ELI-NP will be to extend in the $10 \mathrm{PW}$ regime these experiments, investigating new possibilities to study the properties of the atomic nuclei and of the nuclear forces, taking advantage

Diode-Pumped High Energy and High Power Lasers; ELI: Ultrarelativistic Laser-Matter Interactions and Petawatt Photonics; and HiPER: the European Pathway to Laser Energy, edited by J. Hein, L. O. Silva, G. Korn, L. A. Gizzi, C. Edwards, Proc. of SPIE Vol. 8080, 80800X · @ 2011 SPIE · CCC code: 0277-786X/11/\$18 · doi: 10.1117/12.890139 
mainly on short duration and high-density of laser induced radiation pulses. At $10 \mathrm{PW}$, optical laser pulses focalised to diffraction limit will have an intensity of order of $10^{24} \mathrm{~W} / \mathrm{cm}^{2}$ and maximum electric field strength of $10^{13} \mathrm{~V} / \mathrm{cm}$. One of the main topics of research at ELI-NP will be the generation of brilliant $\gamma$ pulses using, in particular, the inverse Compton back-scattering process to upshift the energy of optical photons in collision with relativistic electrons bunches produced through laser acceleration. In the same time, high-flux and narrow bandwidth $\gamma$ beams will be obtained in laser interaction with classically accelerated electron bunches. With a spectral flux density of $10^{6}$ photons $/ \mathrm{sec} / \mathrm{eV}$, a bandwidth of $0.1 \%$ and an energy variable in 1-19 MeV range, the $\gamma$ beams of ELI-NP will offer unique conditions, compared to other existing $\gamma$ beams facilities, to perform high precision photo-nuclear reaction studies and to develop a wide range of applications.

The parameters of the laser system and of the gamma beam production system are presented in the next section which includes also the facility layout description. In Section 3 the scientific goals are detailed. Section 4 is devoted to the presentation of main application foreseen to be developed at ELI-NP.

\section{ELI-NP FACILITY DESCRIPTION}

The core of ELI-NP facility is a high-power laser system with two parallel amplification chains of $10 \mathrm{PW}$ class. It will use OPCPA technology at the front-end and Ti:Sapphire high-energy amplification stages, similar to the ones developed at the APOLLON laser system[1]. The ELI-NP laser facility will have two front-ends. They will temporally stretch and amplify initial ultrashort pulses with $800 \mathrm{~nm}$ central wavelength to the $100 \mathrm{~mJ}$ level, preserving the needed large bandwidth of the 15 fs laser pulses and the temporal contrast of the pulses in the range of $10^{-12}$. Due to the complexity of such OPCPA system, the alignment and maintenance time for one front-end is long. To avoid such dead-times, one front-end is planned to operate at a time, the second one being used during the maintenance of the other front-end, significantly increasing the available beam-time of the laser facility.

The pulses after the front end are split and distributed to further laser amplifiers, reaching few Joules of energy at $10 \mathrm{~Hz}$ repetition rate and few tens of Joules at a repetition period of the order of few seconds. At these energy levels, the pulses can be extracted from the laser amplification chain and recompressed to shortest duration in vacuum compressors at power of order of $100 \mathrm{TW}$ and respectively $1 \mathrm{PW}$. Subsequently, they are distributed to the high repetition rate experimental areas.

Alternatively, the laser pulses are further amplified in the amplification chains to energies of the order of $200 \mathrm{~J}$. The repetition rate of the pump lasers will restrict the repetition period of the high energy pulses to the minutes range. Adaptive optics and optical isolation of the pulses will be implemented before the optical compressors. After compression, the ultrashort pulses will be distributed to the high energy experimental areas, where standalone experiments or combined experiments using the electron or $\gamma$ beams will be performed. Coherent combination of the two high power ultrashort pulses is envisaged, in order to reach intensities above $10^{23} \mathrm{~W} / \mathrm{cm}^{2}$.

Concerning the gamma beam, ELI-NP electron accelerator will use the X-band technology developed at LLNL for MEGa-ray project [2] but extended from $250 \mathrm{MeV}$ to $600 \mathrm{MeV}$ and a state of the art $10 \mathrm{~J}$ class interaction laser with a repetition rate of $120 \mathrm{~Hz}$ based on diode pumped laser technology. A laser pulse recirculation system synchronized to the trains of electron bunches allows to increase by a factor of 100 the effective gamma pulse rate up to $12 \mathrm{kHz}$. The characteristics of these systems are adapted to produce gamma beams with variable energy up to $19 \mathrm{MeV}, 10^{-3}$ energetic width, $10^{13}$ photons/second total flux and a peak brilliance larger than $10^{21}$ photons $/ \mathrm{sec} / \mathrm{mm}^{2} / \mathrm{mrad}^{2} /(0.1 \% \mathrm{BW})$. After a first stage of acceleration up to $400 \mathrm{MeV}$, a similar laser-electrons interaction system is installed such that intermediary energies gamma beams are available in two additional experimental halls increasing the experiments preparation flexibility and, consequently, the total beam time effectively used. The duration of laser pulses and electron bunches are $2 \mathrm{ps}$, easing the synchronisation with ultrashort high-power lasers pulses required in case of combined experiments.

In Figure 1 the layout of the facility is presented. The laser system up to vacuum compressors is situated in the upper-left area enough large to accommodate future additions of amplification chains. The high-power pulses of the two 
laser arms are transported in parallel to the experimental halls labelled E1, E6 and E7. The high repetition rate pulses of $100 \mathrm{TW} / 1 \mathrm{PW}$ are distributed to halls E4 and E5. The red lines represent the gamma beams. After first interaction point the electron beam is deviated and then steered back in the original direction using symmetric small angle deviations in order to preserve the beam quality. Photonuclear experiments with few $\mathrm{MeV}$ gamma beam will be performed in the E2 and with high energy gamma beams in E8. The E7 hall is devoted to combined experiments. Finally, the E3 area contains the high brilliance positron source placed on the gamma beam axis and the transport system of positron beam at several experimental set-ups to be installed in the upper part of E3 area. Radioprotection will be assured with beam dumps in all relevant locations (including after the laser accelerated beams) and with 1-2 m thick concrete walls. Similar to other nuclear physics facilities, ELI-NP will make extensively use of large concrete blocks to define the experimental halls such that future reconfiguration will be possible.

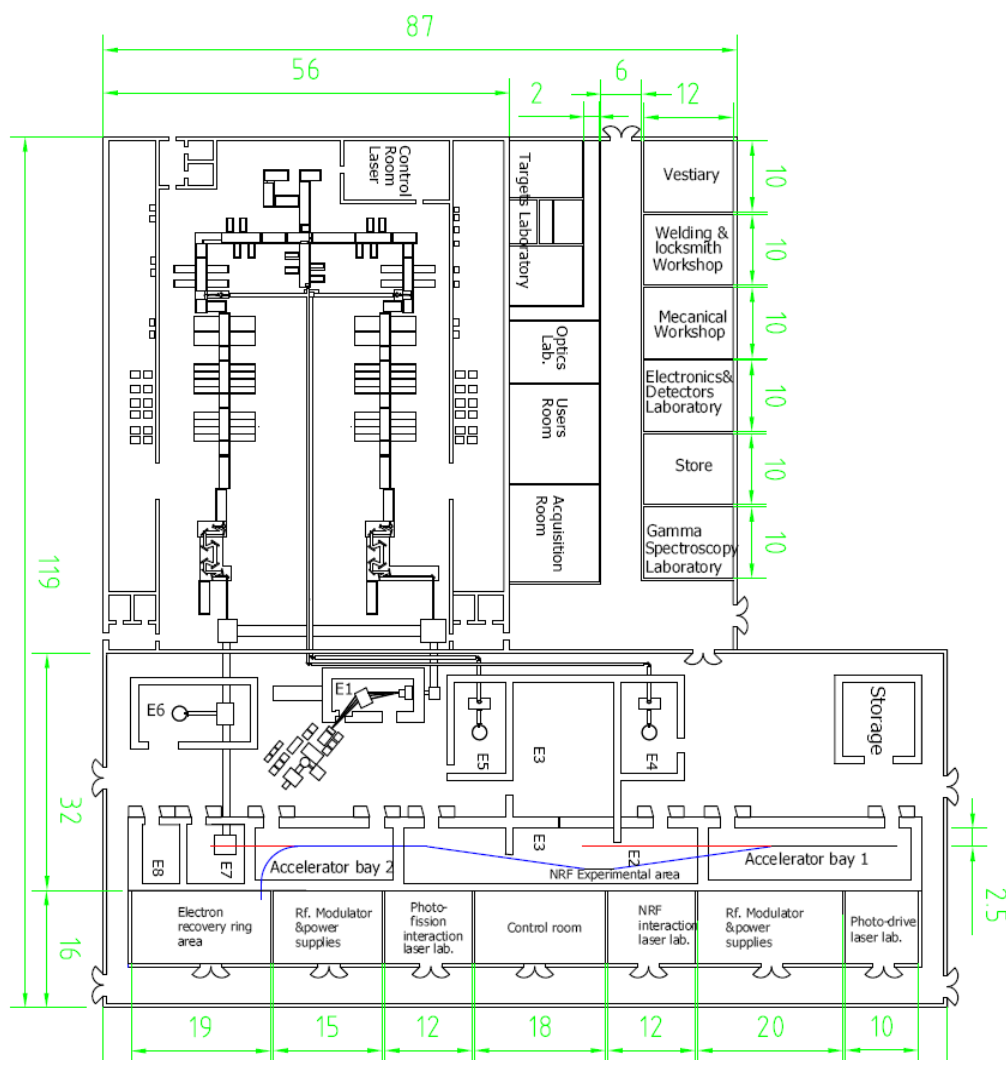

Fig.1 Planned layout of ELI-NP facility.

\section{PHYSICS CASE OF ELI-NP}

This section offers an overview of scientific goals of ELI-NP presented in more details in ELI-NP White Book[3].

Extending the study of ions and electrons acceleration schemes in the $10 \mathrm{PW}$ regime and their optimisation in the directions of interest for nuclear physics experiments are among the main goals of ELI-NP. Characterization of primary and secondary radiation will be carried out with a full range of techniques adapting the instrumentation and methods used currently in nuclear physics such as high-granularity detectors and signal digitisation.

A well established acceleration schemes at laser intensities up to $10^{20} \mathrm{~W} / \mathrm{cm}^{2}$ is the so-called Target Normal Sheath Acceleration e. g. [4], when the electrons are pushed out from the target, form a negative charge cloud and the ions from the target are accelerated by the electrostatic potential created in this way. TNSA is low-efficiency accelerating 
mechanism, where the maximum ion energy scales with the square root of the laser intensity. Recently another acceleration mechanism was experimentally evidenced, the Radiation Pressure Acceleration [5,6]. When a nanometerthick foil is irradiated with circular polarized high intensity laser pulse appears a cold compression of electron sheet followed by the acceleration of ions in the rectified dipole field created between electrons and ions. In this way are created macroscopically neutral bunches of ions and electrons travelling at the same speed. This RPA mechanism is more efficient than TNSA and scales linearly with the laser intensity. The density of accelerated ions in RPA bunch is as high as $10^{22} \mathrm{~cm}^{-3}$ - the solid state density, many orders of magnitude above the classically accelerated ion bunches. This feature was proposed to be exploited [7] in production of the neutron-rich nuclei with $N \cong 126$, a key region of nuclear chart for understanding the heavy elements formation in fast neutron capture nucleosynthesis (r-process).

The high intensity ELI-NP laser pulses will be used to accelerate Thorium nuclei in RPA regime at several $\mathrm{MeV} /$ nucleon. The dense Th bunch will impinge a Thorium secondary target inducing the fission of both in flight and at rest nuclei. The high density of fission products, part of them forward focused with the mean energy of the bunch, could lead to their fusion. As known, the mass distribution of fission fragments has a maximum for the neutron rich nuclei with $\mathrm{A}=80-100$ (light fragments) and a second maximum at $\mathrm{A}=130-150$. The fusion of light fragments is the process of interest for production of neutron rich nuclei with $\mathrm{A}<200$ and $\mathrm{N} \sim 126$. The probability of such interactions could be favourable affected by the predicted decrease of stopping power of high density bunches, meaning that the secondary target could be much thicker. The production rate could be further increased if the above production scheme is assisted by light ions: a fraction of total laser energy could be used to accelerate a large number of light ions from a $\mathrm{CD}_{2}$ foil placed near the first Th target such that to induce the increased number of fissions in the second target and a thin layer of $\mathrm{CD}_{2}$ just in front of the second target will induce the fission of accelerated Thorium before entering in the region were the fusion occurs. The nuclei of interest are expected to exit the target in forward direction allowing separation in magnet spectrometers followed by spectroscopic studies of their decay. In figure 1, the targets and the recoils separator are depicted inside E1 area, the experimental equipments for spectroscopic studies, including a charge breeder and Penning trap for accurate mass measurement are placed outside the heavy shielded area. If such instrumentation is common for many nuclear physics decay spectroscopy experiments, we mentioned that the proposed method for production of radioactive nuclei could give access to $\mathrm{N}=126$ waiting point nuclei with much higher yields than the exiting or next generation of radioactive beams facilities such as FAIR, SPIRAL2 or FRIB.

Another direction of study with high-power lasers at ELI-NP will be the production of brilliant gamma pulses.

The incoherent Thomson scattering of photons on laser accelerated electron bunch have been observed [8] in X-rays domain. The energy $\mathrm{E}_{\gamma}$ of scattered photons, in collinear geometry, is related to electron velocity through the relation $\mathrm{E}_{\gamma}=4 \gamma_{\mathrm{e}}^{2} \mathrm{E}_{0}$ where $\gamma_{\mathrm{e}}=1 /\left(1-\mathrm{v}_{\mathrm{e}} / \mathrm{c}\right)^{1 / 2}$ is the electrons Lorentz factor and $\mathrm{E}_{0} \sim 1 \mathrm{eV}$ is the incoming photons energy. Thus, with electron energies already achieved in laser driven electron acceleration multi-MeV gamma ray will be produced. However, further improvements in energy spread and optical quality of the electron bunches are needed to obtain high brilliance gamma pulses. A promising approach uses the concept of relativistic electron sheet produced by circularly polarized high intensity laser interacting with nanometre foils (see ref. [9] and references therein). The coherent Thomson scattering regime could be reached with high density electron sheets enhancing the gamma production at ELINP to $10^{14} \mathrm{ph} / \mathrm{shot}$ with a pulse duration down to $10^{-21} \mathrm{sec}$ and a brilliance comparable to modern X-ray free electron laser facilities, that is of order of $10^{30}-10^{34} \mathrm{ph} / \mathrm{sec} / \mathrm{mm}^{2} / \mathrm{mrad}^{2} /(0.1 \% \mathrm{BW})$. Such extremely short pulses will open new possibilities to probe nuclear structure and dynamics.

The availability of $\gamma$ beams produced by a warm linac with characteristics orders of magnitude better than any existing facility will provide the ELI-NP facility with a state-of-the-art tool to investigate phenomena and nuclear excitations extremely important for understanding the nuclear structure. Parity doublets, Pigmy and Dipole Resonances, Collective Magnetic States (Scissor Modes) are only a few of the topics studied at ELI-NP.

Nuclear Resonance Fluorescence (NRF) for both fundamental and applied research will be one of the most important subjects to be studied at ELI-NP. The process of NRF corresponds to the excitation of a nuclear state by photons and that state, if it is below the particle threshold, decays by the emission of a photon back to the ground state or 
to another lower excited state. NRF cross sections typically have very large peak values at the energy of that state and correspond to hundreds of barns for excited states in the range of a few $\mathrm{MeV}$.

NRF can be used for many practical applications: nuclear materials, spent fuel rods and radioactive waste can be characterized using the NRF methods. A key nuclear data for the NRF assay is excitation energy and a resonance width of an exited state in nucleus of interest. These nuclear data have not been, however, studied well for long-lived radioisotopes and for those already studied the identification of new excited levels at higher excitation energy could be extremely useful since those levels may provide an enhanced performance in practical systems exploiting NRF to detect special nuclear materials.

On the fundamental topics, NRF with high resolution circularly polarized gamma beam of ELI-NP will provide a sensitive method to measure the parity non-conservation of nucleon-nucleon interaction. The high energy parity doublets in light nuclei such as ${ }^{14} \mathrm{C},{ }^{14} \mathrm{~N},{ }^{15} \mathrm{O},{ }^{16} \mathrm{O},{ }^{18} \mathrm{~F}$ and ${ }^{20} \mathrm{Ne}$ are suggested as best cases for study because the E1/M1 and E2/M2 mixing is expected to be enhanced [10] due to small energy difference in between the doublet members. More general, NRF with a monochromatic gamma beam is the ideal tool to investigate low-lying dipole strength because of selectivity to low spin states that are predominantly excited. Both parts, the electric dipole strength in vibrational nuclei ( $1^{-}$quadrupole-octopole two-phonon states) and magnetic dipole excitation (low lying orbital scissors mode in deformed nuclei, high lying spin-flip mode [11,12]) are very interesting fundamental nuclear physics problems. The Giant Dipole Resonance and Pygmy Dipole Resonance are well known structures of E1 response of nuclei to high energy gamma interpreted, in a hydrodynamic model, as oscillation of neutrons against protons and respectively as neutron skin around $\mathrm{N}=\mathrm{Z}$ core. However their fine structure is still unknown and will become possible to be studied at ELI-NP. Accurate determination of the excitations functions of the gamma induced reactions $(\gamma, n),(\gamma, p),(\gamma, \alpha)$, etc. are also of high interest in astrophysics where such process and their reverse - radiative capture - are the responsible for elements synthesis. All these phenomena can be studied in correlation to deformation or shell effects on stable nuclei or on small (and expensive) targets of low abundance isotopes or even long-lived isomers, taking advantage from ELI-NP high brilliance gamma beam.

Another interesting topic on which the NRF experimental method can shed light is the measurement of the neutron skin of ${ }^{208} \mathrm{~Pb}$. Recently Reinhard and Nazarewicz [13] quantified a strong relationship between the dipole polarizability $\alpha_{\mathrm{D}}$ and the neutron skin of ${ }^{208} \mathrm{~Pb}$ and showed that a precise determination of the neutron radius $\mathrm{r}_{\mathrm{n}}{ }^{\text {rms }}$ with an experimental relative error of $\leq 0.4 \%$ will dramatically reduce theoretical uncertainties in their calculations deriving the neutron matter equation of state (EoS). A model independent measurement of $\alpha_{D}$ will thus allow testing their newly proposed Skyrme-force based functional and help the progress of the Unified Nuclear Energy Density Functional (UNEDF) working group in the effort for a unified description of nucleonic systems from finite nuclei to extended asymmetric systems of astrophysical dimensions such as neutron stars.

Recent developments [14,15] of the Random Matrix Theory and quantum chaos in nuclei will also benefit of orders of magnitude larger ensembles to check their predictions of quantities such as spacing of neighbour levels or the transitions strength distribution. Will become possible to study the transition from collective nuclear motion to chaotic motion on many nuclear species and, based on better understanding of higher laying nuclear level to better predict cross sections of interest for nucleosynthesis.

Additionally, provided that the challenging spatial and temporal synchronization of high-power laser pulses and gamma/ electron bunches is solved, a new experimental window will be open into the largely unexplored domain of nonperturbative quantum electrodynamics (QED) with implications for fundamental issues in quantum field theory, as well as nuclear, atomic, plasma, gravitational and astro-physics.

\section{APPLICATIONS AT ELI-NP}

The range of application using high-power lasers is very broad; however only those related to nuclear physics will be developed at ELI-NP. 
Interaction of the high power ( $>$ PW) laser radiation with the solid state matter produces specific effects, not completely known, on the structure and composition of the irradiated materials. The detailed knowledge of these effects has a fundamental interest for understanding the material behaviour in extreme conditions of irradiation. Tens of fs PW laser pulses and the ultra-short ultra-dense radiation bunches they generate can produce local ultrahigh pressures, of tens Mbar up to Gbar, inside the irradiated materials. These mechanical shocks cause structural defects, compositional inhomogeneities, local melting and fast recrystallization, phase transitions etc. Various samples will be irradiated, such as optical fibres and microelectronic components, and macroscopic effects will be correlated to modification observed at microscopic level using techniques like high-resolution transmission electron microscopy (HRTEM), scanning transmission electron microscopy (STEM), X-ray diffraction (XRD), x-ray photoemission spectroscopy (XPS), electron paramagnetic resonance (EPR) etc.

The following applications are related to the use of gamma rays. These applications will be approached first using the classical accelerator based gamma beams and then with the laser driven acceleration based gamma sources, according the development of such new sources that promise to be more compact and less expenses.

The excitation energies of nuclear states are unique signatures of nuclei which can be exploit for unambiguous detection of their presence. The proposed method [16] is based on NRF process and uses multi-MeV gamma source. Passing through the sample, the gamma rays with the energy corresponding to the transition are absorbed resulting in a depletion (a notch) in the gamma spectrum. The width of the notch is the level width, typically below $1 \mathrm{eV}$ or Doppler width, that is too low to be measured directly. Instead, at the level of a foil containing the isotope of interest placed after the scanned sample, the scattered gamma ray will decrease proportionally to the quantity of searched isotope in the sample. The method benefits from high penetration of gamma ray such that large containers with thick shielding can be scanned. Application are related to security in airports or seaports (search for special nuclear material, toxic substances or explosives), to management of radioactive waste (remote identification of barrels content), to assist the optimal fuel reloading in nuclear power plants through direct measurement of burn-up level of fuel rods.

The nuclear (photo)reactions induced by high-intensity monochromatic gamma beam promise applications with high societal impact: radiopharmaceutical isotope production [17,18]. New production methods are proposed that could yield high specific radioactivity for a number of isotopes with completely new clinical applications of radioisotopes. For example ${ }^{44} \mathrm{Ti}\left(\mathrm{T}_{1 / 2}=59\right.$ a) can be produced via $(\gamma, 2 \mathrm{n})$ reaction and use to generate ${ }^{44} \mathrm{Sc}\left(\mathrm{T}_{1 / 2}=3.9 \mathrm{~h}\right)$ that decay by $\beta^{+}$ followed by a $1157 \mathrm{keV}$ gamma ray. Thus enhancing PET (positron emission tomography) with an additional gamma, a much better spatial resolution could be obtain. Large penetration, submilimeter dimension, low divergence, tuneable energy are features of ELI-NP gamma beams very suitable for radiography and tomography as non-destructive testing application $[19,20]$ of interest for the large-size and complex products, high resolution investigation in aeronautics, automotive, die-cast or sintered industries, new materials and technologies development, for archaeological artifacts and work of art objects analysis.

Using the intense $\gamma$ beam of $10^{13}$ photons/s with energy in the $2.5 \pm 0.5 \mathrm{MeV}$ range and the $\left(\gamma, \mathrm{e}^{+} \mathrm{e}^{-}\right)$reaction, an intense pulsed low energy positron beam of about $10^{7} \mathrm{e}^{+} / \mathrm{s}$ was proposed [21] to be built at ELI-NP. The intensity of this novel source is significantly weaker the presently most intense moderated positron source NEPOMUC [22] at the Munich neutron source FRM 2 with about $9 \cdot 10^{8} \mathrm{e}^{+} / \mathrm{s}$, where about $10^{16} \mathrm{\gamma} / \mathrm{s}$ from neutron capture hit the inner converter volume. However, due to the small diameter and well directed $\gamma$ beam we expect for the new source a brilliance of $2 \cdot 10^{6} \mathrm{e}^{+} /\left[\mathrm{s}(\mathrm{mm} \mathrm{mrad})^{2} 0.1 \% \mathrm{BW}\right]$, which is about higher than the NEPOMUC source. Positron-related techniques offer unique non-destructive methods for materials study, namely in what concerns investigation of defects, band mapping and intimate structure at the surface in the single atomic layer regime. All these techniques have a wide range of technological applications. The positron beam, after extraction from source will transported in E3 large area were several experimental station will be installed for materials investigations using techniques such as: Positron-excited Auger Electron Spectroscopy (PAES), Positron Annihilation Lifetime Spectroscopy (PALS), Coincidence Doppler Broadening Spectroscopy (CDBS) and positron microscopy. 


\section{CONCLUSIONS}

ELI-NP project is the result of a large international collaborative effort, gathering support from individuals and institutions in more than 20 countries from three continents and it aims to be a benefit for the human kind. The contribution of nuclear physics community in defining and enriching the ELI-NP physics case in the form of proposal presented to ELI-NP Workshops and included in ELI-NP White Book is strongly acknowledged. New proposal are welcome, too.

\section{REFERENCES}

[1] J.-P. Chambaret, O. Chekhlov, G. Cheriaux, J. Collier, R. Dabu, P. Dombi, A. M. Dunne, K. Ertel, P. Georges, J. Hebling, J. Hein, C. Hernandez-Gomez, C. Hooker, S. Karsch, G. Korn, F. Krausz, C. Le Blanc, Zs. Major, F. Mathieu, T. Metzger, G. Mourou, P. Nickles, K. Osvay, B. Rus, W. Sandner, G. Szabó, D. Ursescu, K. Varjú, Extreme light infrastructure: laser architecture and major challenges, Proc. SPIE Vol. 7721, 77211D (2010).

[2] Ch. Barty, Development of MEGaRay technology at LLNL, http://www.eli-np.ro/executivecomittee-meeting-april-1213.php (2010).

[3] ELI-NP, http://www.eli-np.ro/documents/ELI-NP-WhiteBook.pdf (2010).

[4] S. C. Wilks, A. B. Langdon, T. E. Cowan, M. Roth, M. Singh, S. Hatchett, M. H. Key, D. Pennington, A. MacKinnon, and R. A. Snavely, Energetic proton generation in ultra-intense laser-solid interactions, Phys. Plasmas $8,542(2001)$.

[5] A.P.L. Robinson, D.-H. Kwon and K. Lancaster, Hole-boring radiation pressure acceleration with two ion species, Plasma Phys. Control. Fusion 51, 095006 (2009).

[6] A. Henig et al., Radiation-Pressure Acceleration of Ion Beams Driven by Circularly Polarized Laser Pulses, Phys. Rev. Lett. 103, 245003 (2009).

[7] D. Habs , P.G. Thirolf, M. Gross, K. Allinger, J. Bin, A. Henig, D. Kiefer, W. Ma, J. Schreiber, Introducing the fission-fusion reaction process: using a laser-accelerated Th beam to produce neutron-rich nuclei towards the $N=$ 126 waiting point of the r-process, Appl Phys B, accepted, arXiv:1007.1251 (2010).

[8] H. Schwoerer et al., Thomson backscattered X-rays from laser accelerated electrons, Phys. Rev. Lett. 96, 014802 (2006).

[9] D. Habs, T. Tajima, J. Schreiber, C.P.J. Barty, M. Fujiwara and P.G. Thirolf, Vision of nuclear physics with photonuclear reactions by laser-driven $\gamma$ beams, Eur. Phys. Jour. D 55, 279 (2009).

[10] A. I. Titov, M. Fujiwara and K. Kawase, Parity non-conservation in nuclear excitation by circularly polarized photon beam, J. Phys. G: Nucl. Part. Phys. 32, 1097 (2006).

[11] U. Kneissl, N. Pietralla, A. Zilges, Low-lying dipole modes in vibrational nuclei studied by photon scattering, J. Phys. G 32, R217 (2006).

[12] K. Heyde, P. von Neumann-Cosel, A. Richter, Magnetic dipole excitations in nuclei: Elementary modes of nucleonic motion, Rev. Mod. Phys. 82, 2365 (2010).

[13] P.-G. Reinhard, W. Nazarewicz, The information content of a new observable: the case of the nuclear neutron skin, Phys. Rev. C 81, 051303(R) (2010).

[14] H.A. Weidenmüller,G.T. Mitchell, Random matrices and chaos in nuclear Physics: Nuclear structure, Rev. Mod. Phys. 81, 539 (2009).

[15] G.E. Michell, A. Richter and H.A. Weidenmüller, Random Matrices and Chaos in Nuclear Physics: Nuclear Reactions, Rev. Mod. Phys. 82, 2845-2901 (2010).

[16] W. Bertozzi, R.J. Ledoux, Nuclear resonance fluorescence imaging in non-intrusive cargo inspection, Nucl. Instrum. Meth. B 241, 820 (2005). 
[17] D. Habs, U. Köster, Production of Medical Radioisotopes with High Specific Activity in Photonuclear Reactions with $\gamma$ Beams of High Intensity and Large Brilliance, Appl. Phys. B, accepted, arXiv-1008.5336v2(2011).

[18] D. Habs, P.G. Thirolf, C. Lang, M. Jentschel, U. Köster, F. Negoita and V. Zamfir, Medical Application Studies at ELI-NP, these proceedings, (2011).

[19] H. Toyokawa et al., Recent progress in generation and application of AIST laser-Compton gamma-ray beam, Nucl. Instr. and Meth. Phys. Res. A 608, S41-S43 (2009).

[20] H. Toyokawa, H. Kanada, T. Kaihori, M. Koike, K. Yamada, Application High-Energy Photon CT System With Laser-Compton Scattering to Non-Destructive Test, IEEE Trans. Nucl. Sci. 55, 3571 (2008).

[21] C. Hugenschmidt, K. Schreckenbach, D. Habs, P. Thirolf, High-Intensity and High-Brightness Source of Moderated Positrons Using a Brilliant $\gamma$ Beam, Appl. Phys. B, submitted, arXiv:1103.0513v1 (2011).

[22] C. Hugenschmidt, K. Schreckenbach, M. Stadlbauer and B. Strasser, Low-energy positron of high intensity at the new positron beam facility NEPOMUC, Nucl. Instr. and Meth. Phys. Res. A 554, 384 (2005). 